\title{
Anthropogenic disturbances consistently favor the high-yield strategists of soil bacterial community in the Eurasian steppe
}

\author{
Yi Fan ${ }^{1,2 \dagger}$, Tingting $\mathrm{Li}^{2 \dagger}$, Wei Liư ${ }^{2 \dagger}$, Minjie Xu ${ }^{2}$, Shuang Pang ${ }^{2}$, Zijia Zhang ${ }^{2}$, Lihua Zhang ${ }^{3}$, Yowhan Son ${ }^{4}$ and \\ Ximei Zhang ${ }^{2^{*}}$ (D)
}

\begin{abstract}
Purpose: Multiple anthropogenic disturbances, such as climate warming and nitrogen deposition are affecting terrestrial ecosystems. Different disturbances may have some consistent effects on the soil microbial community, which remains largely unexplored.

Methods: We mimicked 16 anthropogenic disturbances in a steppe ecosystem, and measured the absolute abundance and taxonomic composition of soil bacterial communities with qPCR and amplicon sequencing, respectively.

Results: We found that while the absolute abundance of each of the four dominant bacterial phyla did not show a consistent response to these disturbances, that of the five subdominant phyla showed a consistent increase. Meanwhile, these disturbances consistently stimulated the relative abundances of metabolic functions for high-growthyield, including the transport/metabolism of amino acids and carbohydrates. Stochastic processes (e.g., random birth) played more critical roles in structuring the subdominant than dominant phyla, and the disturbances promoted the stochastic processes.

Conclusions: Overall, the high-yield traits and stochasticity of subdominant phyla led to their positive responses to disturbances. Furthermore, our findings indicate that the intensifying human activities are likely to cause a high-yieldstrategies-toward shift in soil microbial composition in the Eurasian steppe ecosystem.
\end{abstract}

Keywords: Disturbance, Grassland, Metagenome, Stochasticity

Multiple environmental changes affect terrestrial ecosystems globally, including soil microbial communities, which harbor perhaps the highest microbial species diversity on Earth (Rappe and Giovannoni 2003). However, it remains largely unexplored whether different environmental changes have some consistent effects on soil microbial communities. All environmental changes

\footnotetext{
*Correspondence: zhangximei@caas.cn

${ }^{\dagger}$ Yi Fan, Tingting Li, and Wei Liu contributed equally to this article.

${ }^{2}$ Key Laboratory of Dryland Agriculture, Ministry of Agriculture, Institute

of Environment and Sustainable Development in Agriculture, Chinese

Academy of Agricultural Sciences, Beijing 100081, China

Full list of author information is available at the end of the article
}

have in common that they disrupt the originally stable soil environment. In other words, all environmental changes act as disturbance events (Ferrenberg et al. 2013). Thus, they may consistently promote community re-assembly by mediating the stochastic ecological processes such as random population dynamics (i.e., birth, death) and species colonization until the environment reaches a new equilibrium point (Zhang et al. 2016a; Zhou and Ning 2017). Meanwhile, different microbial groups survive in the soil by adopting different life history strategies (Fierer et al. 2007; Malik et al. 2020). For example, resource-acquisition-strategists invest a large 
proportion of acquired resources to produce extracellular enzymes to break down complex substrates, and they also exhibit competitor traits such as antibiotic production and defense. The high-yield-strategists allocate a lot of resources to biosynthetic processes, such as amino acid, fatty acid, and nucleotide synthesis. Since different disturbances consistently alter the soil environment, which means the creation and release of some new resources/space (Zhang et al. 2016a), we hypothesize that they will consistently stimulate the high-yield-strategists of a microbial community through promoting stochastic ecological processes.

To test this hypothesis, we conducted four 5-year multi-factorial experiments in a steppe ecosystem in Inner Mongolia, China, which is floristically and ecologically representative of much of the Eurasian steppe region ( $\mathrm{Li}$ et al. 1988). The 4 experiments mimicked a total of 16 anthropogenic disturbances, including the removal of one, two, or three plant functional groups, mowing, nitrogen addition, phosphorus addition, water addition, warming, and several of these treatments in combination (mowing plus nitrogen addition, mowing plus phosphorus addition, mowing plus watering, nitrogen addition plus phosphorus addition, nitrogen addition plus watering, watering plus warming, mowing plus nitrogen addition plus phosphorus addition, mowing plus nitrogen addition plus watering; Method S1, Table S1, and Table S2) (Zhang et al. 2016a). We investigated the absolute abundance and taxonomic composition of soil bacteria with qPCR and 454 pyrosequencing, respectively. The DNA sequence composition of the microbial community was measured with HiSeq 2000 shotgun metagenomic sequencing to obtain the information of all genes of all soil microbial species. Briefly, DNA extracts were processed according to the description of the Illumina Paired-End Prep kit protocol. DNA was sheared mechanically and sizeselected to $\sim 180$-bp and gel purified. Sequencing was performed in an Illumina Hiseq 2000 platform located at Shanghai Majorbio Bio-pharm Technology Co., Ltd. $2.18 \pm 0.08$ (mean \pm SE) Giga base pairs of DNA sequences were acquired for each sample (see details in Method S1).

The relative abundances of ten common bacterial phyla quantified with 454 pyrosequencing were strongly correlated with those determined with metagenomic sequencing $(P<0.001$; Fig. S1), demonstrating the high consistency between these complementary approaches and the reliability of the relative abundance results. Thus, the absolute abundance of each phylum was calculated as the product of its relative abundance from 454 pyrosequencing and the absolute abundance of the entire bacterial community from qPCR. The effect size of each phylum under each disturbance (Table S3) is calculated as ((its mean absolute abundance under environmental change)-(its mean absolute abundance under control))/(its mean absolute abundance under control). The ten bacterial phyla were ordered by their relative abundances, from the highest to the lowest. For each of the first four phyla (Actinobacteria, Proteobacteria, Acidobacteria, and Chloroflexi), the effect sizes were not significantly different from zero across all 16 disturbances $(P>0.05$; Fig. 1a). In contrast, for each of the subsequent five phyla (Bacteroidetes, Gemmatimonadetes, Firmicutes, Nitrospirae, and Planctomycetes), the effect sizes were significantly $>0$ across the 16 disturbances $(P<0.05$; Fig. 1b). For convenience, the first four phyla were taken as the dominant phyla, and the subsequent five phyla were taken as the subdominant phyla. Overall, these results meant that different disturbances consistently stimulated the five subdominant phyla. The soil physicochemical indices alone could not explain this pattern (Data S1; Table S4).

The COG (Clusters of Orthologous Groups of proteins) categories were used to represent the major metabolic functions of microbial communities. The relative abundance of each category was estimated by mapping the metagenomic reads against the protein sequence of the STRING database (von Mering et al. 2003). Similar to the effect size of phylum absolute abundance, we also calculated the effect size of each COG category basing on its relative abundance (Table S5). Among all the 25 COG categories, there were 6 with effect sizes significantly $>0$ across the 16 disturbances, including the categories responsible for the transport/metabolism of amino acid, carbohydrate, coenzyme, and lipid (Fig. 1b). These functions are often related to the high-yield traits (Malik et al. 2020), suggesting that the five subdominant phyla with positive effect sizes are primarily high-yield-strategists. Accordingly, there were some categories with effect sizes consistently $<0$, such as those responsible for defense mechanisms and extracellular structure (Fig. 1b), which were indicative of the resource-acquisition traits (Malik et al. 2020). These disturbances increased the absolute abundances of the five subdominant phyla, and accordingly, decreased the relative abundances of other phyla in the entire communities (e.g., the dominant phyla, although their absolute abundances did not change). In other words, the four dominant phyla are likely to be resource-acquisition-strategists.

We further calculated the SES (standard effect size) value with a null model method to estimate the effect 

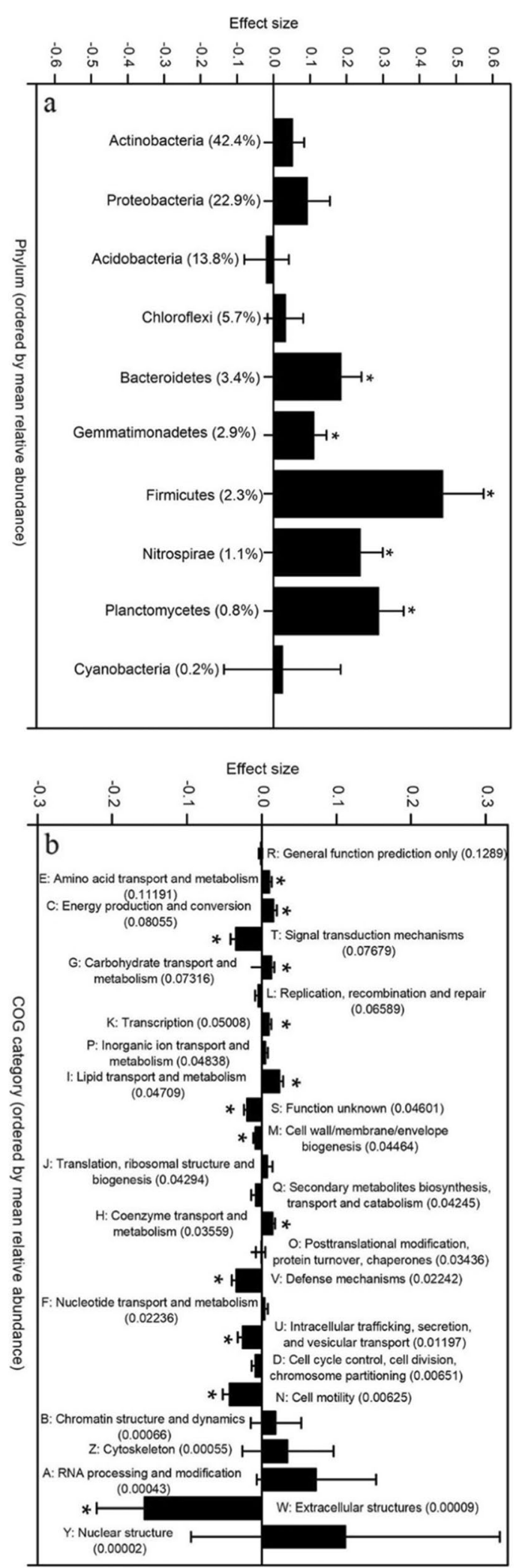

Fig. 1 The effect size of each microbial phylum (a) or function (b) in the grassland ecosystem under investigation. In b, the functional name is the COG category abbreviation (the capital) and the functional description. The effect size of each phylum under each environmental change is calculated as: (its mean absolute abundance under environmental change) - (its mean absolute abundance under control))/(its mean absolute abundance under control). The relative abundance is used for the calculation of the effect size of each function. See the detailed value of effect size of each phylum/function under each environmental change in Table S3 and S5. For each phylum/function, the mean and standard error of the effect sizes under 16 environmental changes are shown in the figure. The percentage in the brackets following the phylum/ function name is its mean relative abundance in the entire bacterial community. ${ }^{*}$ denotes instances when the effect size was significantly different from zero (one sample $T$ test: $P<0.05$ )

of deterministic relative to stochastic processes on community assembly (Chase 2010). The SES values were significantly smaller for the subdominant than the dominant phyla $(P<0.05$; Fig. 2$)$, indicating that the subdominant phyla were more stochastic than the dominant phyla. Similarly, stochastic processes were found to play more critical roles in driving the dynamics of low-abundance species than those of highabundance species in plant communities (Zhang et al. 2016b). Furthermore, the SES values were significantly smaller for the disturbance conditions than the control $(P<0.05$; Fig. 2$)$, suggesting that the disturbances promoted the stochastic processes further.

In summary, the five subdominant phyla had highyield traits, and stochastic processes played important roles in structuring them. Environmental changes serving as disturbance events promoted the stochastic processes and thus, caused a consistent increase in the absolute abundance of these high-yield-strategists, supporting our hypothesis. In contrast, the four dominant phyla had resource-acquisition traits, were structured primarily by deterministic processes, and different disturbances had no consistent effect. It should be noted that our results indicated only the overall high-yield-versus resource-acquisition traits of each phylum, but did not imply that all species with a phylum had the same trait. Our findings imply that the intensifying human activities have already caused and will continue to cause a systematically high-yield-strategist-toward shift in the soil microbial composition in the Eurasian steppe ecosystem and even in many other ecosystems on the Earth. In addition, these disturbances had no consistent effect on the absolute abundance of the phylum Cyanobacteria, the 10th abundant phyla in this study, either (Fig. 1a). This phylum (and perhaps many others with even lower abundances) 


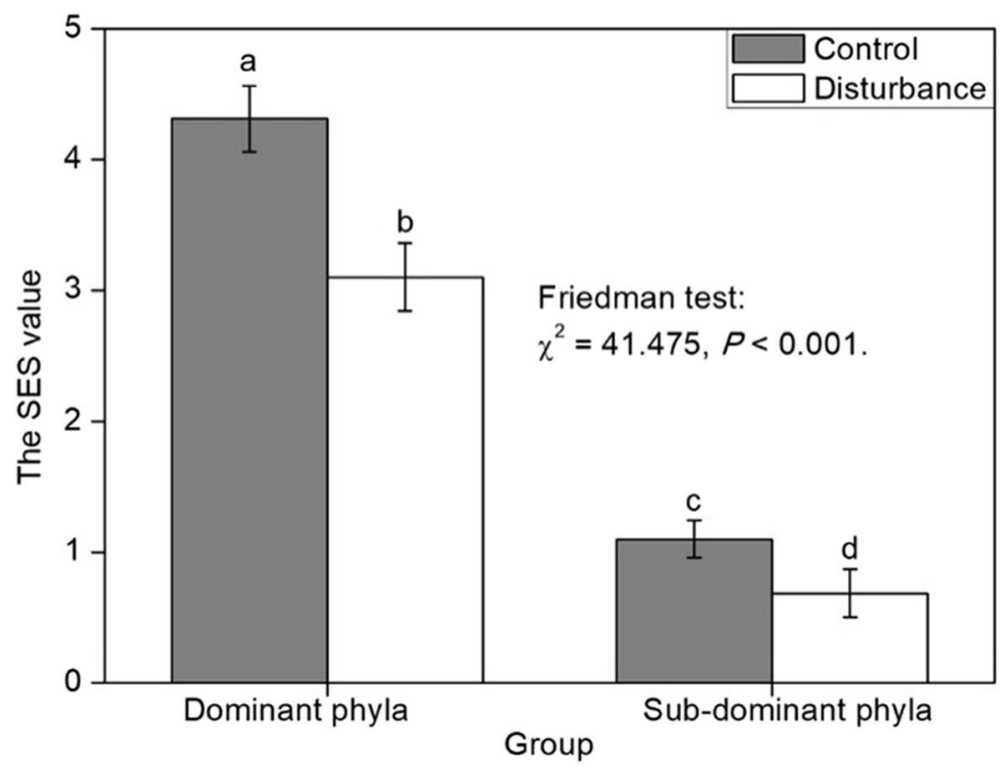

Fig. 2 The SES value for the four dominant and five subdominant bacterial phyla under the control and disturbance conditions. The value of SES (standard effect size) is calculated with a null model method, and it represents the magnitude of deterministic relative to stochastic processes in the process of community assembly. The mean and standard error of SES values under 16 conditions are shown. The different letters (from a to d) above the four groups meant that there were significant differences (Friedman test: $P<0.05$ ) in the SES value between them

might belong to neither the high-yield-strategists nor the resource-acquisition-strategists. However, it acts as stress-tolerators, as stated in the Grimes competitor-stress tolerator-ruderal (CSR) framework (Fierer 2017; Malik et al. 2020), which needs to be examined in the future.

\section{Supplementary Information}

The online version contains supplementary material available at https://doi. org/10.1186/s13213-021-01658-0.

Additional file 1: Data S1. Effect of soil physicochemical indices on bacterial phylum abundance.

Additional file 2: Figure S1. The positive correlation between the relative abundances of bacterial phyla quantified with 454 sequencing and those quantified with HiSeq 2000 metagenome sequencing.

Additional file 3: Method S1. Supporting materials and methods.

Additional file 4: Table S1. Plant functional groups and their properties. Table S2. Design of the first experimental part. Table S3. The effect size of 10 relatively dominant bacteria phyla under each of the 16 environmental changes. Table S4. The effect size of soil physicochemical indices under each of the 16 environmental changes. Table S5. The effect size of each COG category under each of the 16 environmental changes.

\section{Acknowledgements}

We thank Dr. Paul Kardol and Eric R. Johnston for their help in polishing of the language.

\section{Authors' contributions}

$Y F, T L$, and WL carried out the experiment. XZ designed the research and wrote the manuscript. All authors have read and approved the final manuscript

\section{Funding}

This research was supported by Key Laboratory of Soil Environment and Nutrient Resources of Shanxi Province (to Ximei Zhang), National Natural Science Foundation (32071547) and Central Public-interest Scientific Institution Basal Research Fund (No. BSRF202106) of China.

\section{Availability of data and materials}

All data analyzed during this study are included in this published article and its supplementary information files.

\section{Declarations}

Ethics approval and consent to participate

Not applicable.

\section{Consent for publication}

Not applicable.

\section{Competing interests}

The authors declare that they have no competing interests.

\section{Author details}

${ }^{1}$ Key Laboratory of Soil Environment and Nutrient Resources of Shanxi Province, Taiyuan 030031, China. ${ }^{2}$ Key Laboratory of Dryland Agriculture, Ministry of Agriculture, Institute of Environment and Sustainable Development in Agriculture, Chinese Academy of Agricultural Sciences, Beijing 100081, China. ${ }^{3}$ College of Life and Environmental Sciences, Minzu University of China, Beijing 100081, China. ${ }^{4}$ Division of Environmental Science and Ecological Engineering, Korea University, Seoul 02841, Korea. 
Received: 26 August 2021 Accepted: 15 November 2021

Published online: 28 November 2021

\section{References}

Chase JM (2010) Stochastic community assembly causes higher biodiversity in more productive environments. Science 328:1388-1391

Ferrenberg S, O'Neill SP, Knelman JE, Todd B, Duggan S, Bradley D, Robinson T, Schmidt SK, Townsend AR, Williams MW, Cleveland CC, Melbourne BA, Jiang L, Nemergut DR (2013) Changes in assembly processes in soil bacterial communities following a wildfire disturbance. ISME J 7:1102-1111

Fierer N (2017) Embracing the unknown: disentangling the complexities of the soil microbiome. Nat Rev Microbiol 15:579-590

Fierer N, Bradford MA, Jackson RB (2007) Toward an ecological classification of soil bacteria. Ecology 88:1354-1364

Li B, Yong S, Li Z (1988) The vegetation of the Xilin River basin and its utilization. In: Inner Mongolia Grassland Ecosystem Research Station (ed). Research on Grassland Ecosystem No.3. Science Press, Beijing, pp 84-183

Malik AA, Martiny JBH, Brodie EL, Martiny AC, Treseder KK, Allison SD (2020) Defining trait-based microbial strategies with consequences for soil carbon cycling under climate change. ISME J 14:1-9

Rappe MS, Giovannoni SJ (2003) The uncultured microbial majority. Annu Rev Microbiol 57:369-394

von Mering C, Huynen M, Jaeggi D, Schmidt S, Bork P, Snel B (2003) STRING: a database of predicted functional associations between proteins. Nucleic Acids Res 31:258-261

Zhang X, Johnston ER, Liu W, Li L, Han X (2016a) Environmental changes affect the assembly of soil bacterial community primarily by mediating stochastic processes. Glob Chang Biol 22:198-207

Zhang X, Pu Z, Li Y, Han X (2016b) Stochastic processes play more important roles in driving the dynamics of rarer species. J Plant Ecol 9:328-332

Zhou J, Ning D (2017) Stochastic community assembly: does it matter in microbial ecology? Microbiol Mol Biol R 81:e00002-e00017

\section{Publisher's Note}

Springer Nature remains neutral with regard to jurisdictional claims in pub-

lished maps and institutional affiliations.

- fast, convenient online submission

- thorough peer review by experienced researchers in your field

- rapid publication on acceptance

- support for research data, including large and complex data types

- gold Open Access which fosters wider collaboration and increased citations

- maximum visibility for your research: over $100 \mathrm{M}$ website views per year

At BMC, research is always in progress.

Learn more biomedcentral.com/submissions 